IOS Press

\title{
Phenolic compounds profile of strawberry fruits of Charlotte cultivar
}

\author{
Agnieszka Kosińska ${ }^{\mathrm{a}, \mathrm{b}, *}$, Sascha Diering ${ }^{\mathrm{a}, \mathrm{c}}$, Denis Primª ${ }^{\mathrm{a}}$, Julien Héritier ${ }^{\mathrm{a}}$ and Wilfried Andlauer ${ }^{\mathrm{a}}$ \\ ${ }^{a}$ Institute of Life Technologies, University of Applied Sciences Valais, Sion, Switzerland \\ ${ }^{\mathrm{b}}$ Division of Food Science, Institute of Animal Reproduction and Food Research of the Polish Academy of Sciences, \\ Olsztyn, Poland \\ ${ }^{\mathrm{c}}$ Department of Nutrition and Food Sciences, University of Bonn, Bonn, Germany
}

Received 13 June 2012; accepted 15 September 2012

\begin{abstract}
The profile of phenolic compounds in strawberry fruits varies significantly among cultivars. High performance liquid chromatography with diode array detection coupled to electrospray ionization mass spectrometric detection with positive and negative modes of ionization was employed to identify phenolic compounds in extract of strawberries from Charlotte cultivar. This is the first time phenolic profile of Charlotte cultivar has been characterized. The fruits contained phenolics belonging to six groups: anthocyanins, ellagic acid and its conjugates, gallotannins, flavonols, flavanols and hydroxycinnamic acid derivatives. The presence of pentagalloyl glucose in strawberries fruits was reported for the first time.
\end{abstract}

Keywords: Strawberries, phenolic compounds, Charlotte cultivar, HPLC-DAD-ESI-MS/MS

\section{Abbreviations}

$\begin{array}{ll}\text { HHDP } & \begin{array}{l}\text { hexahydroxydiphenoyl } \\ \text { HPLC-DAD-ESI-MS/MS }\end{array} \\ & \begin{array}{l}\text { high performance liquid chromatography diode array detection electrospray } \\ \text { ionization mass spectrometry } \\ \text { molecular weight }\end{array} \\ \text { MW } & \text { mass to charge ratio } \\ \text { m/z } & \text { retention time } \\ \text { RT } & \text { strawberry fruit extract } \\ \text { SE } & \end{array}$

\section{Introduction}

Berries contain high amount of phytochemicals, mainly phenolic molecules which exhibit wide range of activity [1-4]. Strawberries are commonly consumed worldwide, mainly as fresh fruits. Their anti-inflammatory properties, the ability to modulate cellular signaling pathways of cancer cell proliferation, apoptosis and tumor angiogenesis and the role in the diminishing the risk of cardiovascular disease have been reported [5-7]. In order to assess potential health effects of fruits, their phenolic compounds profile should be identified. The content of phenolic compounds in strawberry fruits is affected by various factors such as cultivar, agricultural practice, environment, and ripening stage [8]. A wide range of strawberry cultivars are grown around the world. The studies conducted on 27 cultivars from Norway [9], 20 from Spain [10, 11], 3 from Turkey [12], and 4 from Macedonia [13] revealed

*Corresponding author: Agnieszka Kosińska, Tel.: +48 89 5234627; Fax: +48 89 5240124; E-mail: a.kosinska@pan.olsztyn.pl. 
large differences in phenolics composition and antioxidant activity of strawberry fruits between cultivars. A recently published paper indicated that genotype was a major determining factor of bioactive components [14]. Currently the efforts of breeding programs are focused on developing new cultivars with enhanced flavour and preferably also improved levels of health-beneficial compounds $[15,16]$. Charlotte variety was created in a breeding program by crossing 'Mara des Bois' by 'CAL 19' [17]. The selection of the variety was mainly based on the fruits of good quality, desirable taste, disease tolerance and everbearing, which allows a consistent production during the year. The characteristic feature of fruits of this cultivar is their interesting aroma profile and sweet taste due to high levels of fructose and glucose [16].

Nowadays, knowledge of bioactivity of individual phenolic compounds is being expanded. The more we know about beneficial effect of phenolic compounds, the more important becomes the analysis of phenolic profiles of fruits of different varieties. Phenolic profile, along with vitamin C content, helps to identify fruits of good quality, exhibiting health promoting properties. The present study investigated phenolic compound profile of Charlotte cultivar, which has not been reported up to date.

\section{Materials and methods}

\subsection{Chemicals and standards}

Acetone and ethanol (absolut) were purchased from Cochimy (Martigny, Switzerland). Formic acid and Amberlite XAD-16 were obtained from Sigma-Aldrich (Buchs, Switzerland). Acetonitrile of HPLC grade were acquired from Lab-Scan (Gliwice, Poland).

\subsection{Plant material}

Strawberries of Charlotte cultivar were obtained from ACW research centre (Agroscope Changins-Wädenswil, Switzerland). Strawberries were cultivated under plastic tunnel of $8 \mathrm{~m}$ in width $25 \mathrm{~m}$ in length (made of $200 \mu \mathrm{m}$ thick ethylene-vinyl acetate copolymers) on TopferdeKF (Ökohum) composed of: $48 \%$ white peat and $52 \%$ of bark and fibre of coconut, characterised by water retention of $600 \mathrm{ml} / \mathrm{l}, \mathrm{pH}$ of 5.5 and conductivity of $0.3 \mathrm{mS} / \mathrm{cm}$, which is an reference substrate for strawberries cultivation in Switzerland. The fruits were harvested at commercial ripeness and frozen immediately at $-20^{\circ} \mathrm{C}$.

\subsection{Extraction procedure}

A portion of 1,000 g of fruits was ground using a Büchi Mixer B-400 (Büchi, Flawil, Switzerland) applying a rotation speed of 9,000 rpm for 3-5 s. The obtained puree was freeze-dried (Lyolab B II, LSL Secfroid, Switzerland). The extraction of phenolic compounds was performed as described by Kosińska et al. [18]. Briefly, a solvent mixture consisting of acetone, water and formic acid $(70 / 29.9 / 0.1, \mathrm{v} / \mathrm{v} / \mathrm{v}, \mathrm{pH}=4.02)$ was mixed with strawberry powder at a solid to solvent ratio of $1: 12$. Then, the homogenization was performed with an Ultra Turrax T25 (Ika Labortechnik, Staufen, Germany) at 20,500 rpm for $3 \mathrm{~min}$, the extract was centrifuged at 5,000 $\mathrm{g}$ and $5^{\circ} \mathrm{C}$ for 10 min (Universal 32R, Hettick Zentrifugen), the supernatant was collected and the precipitate was homogenized with the solvent again. The procedure was repeated twice. The solvent from combined extracts was evaporated immediately using a rotary evaporator (Heidolph Instruments $\mathrm{GmbH} \&$ Co. KG, Schwabach, Germany). The extract was purified chromatographically on a column filled with Amberlite XAD-16. Sugars and organic acids were removed with water, whereas phenolic compounds were eluted with aqueous ethanol, and solvent was evaporated at $40^{\circ} \mathrm{C}$.

\subsection{LC-DAD-ESI-MS/MS analysis}

An Agilent 1200 series liquid chromatograph (Agilent Technologies, CA, USA) comprised of an autoinjector, a quaternary pump, a column counterpart and an UV-DAD detector. The strawberry purified extract (SE) was dissolved in methanol/water/formic acid mixture (50/49.9/0.1, v/v/v) at a concentration of $5 \mathrm{mg} / \mathrm{ml}$ and filtered through a $13 \mathrm{~mm}$ 
PTFE filter with a pore size of $0.45 \mu \mathrm{m}$ (EXAPURE ${ }^{\mathrm{TM}}$, Switzerland). The volume of $20 \mu \mathrm{l}$ was injected onto a Luna $5 \mu \mathrm{C} 18$ (2) column $(250 \times 4.6 \mathrm{~mm}$ i.d., particle size $5 \mu \mathrm{m}$, Phenomenex, Torrance, CA, USA) with a C18 security guard column $(4 \times 2.0 \mathrm{~mm}$ i.d., $5 \mu \mathrm{m}$, Phenomenex $)$ at a flow rate of $0.7 \mathrm{ml} / \mathrm{min}$. The mobile phase was composed of $0.1 \%$ aqueous formic acid water (A) and acetonitrile containing $0.1 \%$ formic acid (B). A linear gradient was performed as follows: $10-18 \% \mathrm{~B}$ (0-10 min); 18\% B (10-15 min); 18-25\% B (15-30 min); 25-85\% B (30-31 min); $85 \%$ B (31-40 min). The column was re-equilibrated between injections for 10 min with $90 \%$ of mobile phase B. The column temperature was set at $30^{\circ} \mathrm{C}$. Spectral data for all peaks were collected in the range of 200-600 nm and separation was recorded at 260, 280, 360 and $500 \mathrm{~nm}$. Standard solutions of ellagic acid, $p$-coumaric acid, pelargonidin-3-glucoside and cyanidin-3-glucoside were used for identification.

The mass spectra were acquired using a ThermoQuest-Finningan LCQ DECA controlled by Xcalibur 2.0 software. The mass spectra were acquired using electrospray ionisation in the negative and positive ionisation modes in the range of $\mathrm{m} / \mathrm{z} 100-2,000$. The capillary temperature was set at $275^{\circ} \mathrm{C}$, sheath gas flow was 60 arbitrary units. The source voltage was set at $4 \mathrm{kV}$. Collision induced dissociation fragmentation was performed.

\section{Results and discussion}

\subsection{Identification of phenolic compounds}

The HPLC-DAD profiles of phenolic compounds of Charlotte SE recorded at 280, 360 and $500 \mathrm{~nm}$ were depicted in the Fig. 1. Retention time (RT), maximum absorption wavelength $\left(\lambda_{\max }\right)$, molecular ions and fragment ions masses of identified compounds were compiled in Table 1. Analysis of obtained data was accompanied by its comparison with literature data on phenolic compounds of strawberries of different cultivars $[9,11,13,19,20]$. The SE contained phenolic compounds belonging to six main groups: anthocyanins, ellagic acid and its conjugates, hydroxycinnamic acid derivatives, gallotannins, flavonols and flavanols.

\subsection{Anthocyanins}

Anthocyanin pigments are responsible for the attractive red, purple to blue colours of many fruits and vegetables. They have a typical absorption band in the 490 to $550 \mathrm{~nm}$ region of the visible spectra. The chromatographic profile of anthocyanins present in SE was presented in Fig. 1C. Cyanidin-3-glucoside (peak 8) and pelargonidin-3-glucoside (peak 9) were identified by comparing their RT and UV spectra with those of corresponding commercial standards, and their structures were confirmed by HPLC-ESI-MS. Due to observed tailing of peak 9 and appearance of an ion at a $\mathrm{m} / \mathrm{z}, 579$ together with an ion characteristic for pelargonidin-3-glucoside $(\mathrm{m} / \mathrm{z}$ of 433$)$ it might be supposed that pelargonidin-3-rutinoside is co-eluting in peak 9. Those compounds have been reported to elute with similar RT [11, 13]. The compound eluting at a RT of 5.3 min (peak 3) was identified as pelargonidin-3-dihexose since it displayed an ion at a $\mathrm{m} / \mathrm{z}$ of 595 in positive mode of ionization. The fragmentation of this ion resulted in two ions at $\mathrm{m} / \mathrm{z}$ of 433 (loss of hexose) and 271 (aglycone of pelargonidin). Most probably it is pelargonidin-3,5-diglucoside which was previously identified in strawberries [11]. The fragment ion at a $\mathrm{m} / \mathrm{z}$ of 271 was also present in the mass spectra of peak 17, suggesting the presence of pelargonidin derivative. Due to its molecular ion at $\mathrm{m} / \mathrm{z}$ of 519 it was identified as pelargonidin-3-malonylglucoside. The small peak eluting at 12.67 min displayed a molecular ion at a $\mathrm{m} / z$ of 535 and a fragment ion at a $\mathrm{m} / \mathrm{z}$ of 287 , which corresponds to cyanidin-3-malonylglucoside.

\subsection{Ellagic acid and its conjugates}

On the basis of UV spectra and comparison of the RT to that observed for standard compound, peak 28 was identified as ellagic acid, which was confirmed by mass spectra analysis. Peaks 22 and 25 displayed a fragment ion at a $m / z$ of 301, which corresponds to ellagic acid. The negative molecular ion at $m / z$ of 433 and 447 , respectively, were noticed. The loss of $132 \mathrm{Da}$ may correspond to a pentose, whereas the loss of 146 to a deoxyhexoside, which led to identification of peaks 22 and 25 as ellagic acid pentoside and ellagic acid deoxyhexoside, respectively. 
Table 1

Absorption maximum in the UV-VIS region $\left(\lambda_{\max }\right)$, mass spectral data, and identification of phenolic compounds of strawberry fruits of Charlotte cultivar

\begin{tabular}{|c|c|c|c|c|c|c|c|}
\hline Peak & $\mathrm{RT}(\min )$ & $\lambda_{\max }(\mathrm{nm})$ & $\operatorname{MS}(m / z)$ & $\operatorname{MS}^{2}(m / z)$ & Identified compound & MW & Reference \\
\hline 1 & 3.97 & 242 & $175[\mathrm{M}-\mathrm{H}]^{-}$ & 115 & Ascorbic acid & 176 & {$[9,20]$} \\
\hline 2 & 5.31 & 282 & $865[\mathrm{M}-\mathrm{H}]^{-}$ & $739, \mathbf{6 9 5}, 577$ & Procyanidin trimer & 866 & {$[9,13,20]$} \\
\hline 3 & 5.30 & 510,280 & $595[\mathrm{M}]^{+}$ & $\mathbf{4 3 3}, 271$ & Pelargonidin-3-diglucoside & 595 & {$[11,13,19]$} \\
\hline 4 & 7.02 & 274 & $783[\mathrm{M}-\mathrm{H}]^{-}$ & $481, \mathbf{3 0 1}, 275$ & di-HHDP-glucose & 784 & {$[13,20]$} \\
\hline 5 & 7.62 & 242 & $783[\mathrm{M}-\mathrm{H}]^{-}$ & $481, \mathbf{3 0 1}, 275$ & di-HHDP-glucose & 784 & {$[13,20]$} \\
\hline 6 & 8.15 & 279 (broad), 221 & $205[\mathrm{M}+\mathrm{H}]^{+}$ & 188 & Tryptophan & 204 & [27] \\
\hline 7 & 9.19 & 242 & $1917[\mathrm{M}-\mathrm{H}]^{-}$ & $1833,1531, \mathbf{1 0 5 3}, 883$ & unknown & & \\
\hline 8 & 8.25 & 515,278 & $449[\mathrm{M}]^{+}$ & 287 & Cyanidin-3-glucoside & 449 & {$[2,9,13,19,20]$} \\
\hline \multirow[t]{2}{*}{9} & 9.90 & 500,276 & $433[\mathrm{M}]^{+}$ & 271 & Pelargonidin-3-glucoside & 433 & {$[9,13,19,20]$} \\
\hline & & & $579[\mathrm{M}]^{+}$ & 433,271 & Pelargonidin-3-rutinoside & 579 & {$[2,13]$} \\
\hline 10 & 10.47 & 286 & $449[\mathrm{M}-\mathrm{H}]^{-}$ & $\mathbf{3 5 5}, 325,287,269,193$ & Ferulic acid hexose derivative & 450 & {$[9,13,20]$} \\
\hline 11 & 11.12 & 280 & $577[\mathrm{M}-\mathrm{H}]^{-}$ & $431, \mathbf{4 2 5}, 407,289$ & Procyanidin dimer & 578 & {$[9,13,20]$} \\
\hline 12 & 11.28 & 337,220 & $633[\mathrm{M}-\mathrm{H}]^{-}$ & $463, \mathbf{3 0 1}$ & HHDP-galloyl-glucose & 634 & {$[13,20]$} \\
\hline 13 & 11.55 & 280 & $865[\mathrm{M}-\mathrm{H}]^{-}$ & $739, \mathbf{6 9 5}, 577,407$ & Procyanidin trimer & 866 & {$[9,13,20]$} \\
\hline 14 & 12.35 & 315,228 & $325[\mathrm{M}-\mathrm{H}]^{-}$ & $265,187,163,145$ & $p$-Coumarylhexose & 326 & {$[9,13,19,20]$} \\
\hline 15 & 12.67 & 512,312 & $535[\mathrm{M}]^{+}$ & 287 & Cyanidin-3-malonylglucoside & 539 & {$[2,9,13]$} \\
\hline 16 & 13.34 & 284 & $1441[\mathrm{M}-\mathrm{H}]^{-}$ & $1315,865, \mathbf{5 7 5}$ & Procyanidin pentamer & 1442 & [9] \\
\hline 17 & 14.51 & 502,277 & $519[\mathrm{M}]^{+}$ & 271 & Pelargonidin-3-malonylglucoside & 519 & {$[2,9,13,20]$} \\
\hline 18 & 15.65 & 344 sh, 281 & $449[\mathrm{M}-\mathrm{H}]^{-}$ & $\mathbf{3 5 5}, 287,269$ & Ferulic acid hexose derivative & 450 & {$[9,13,20]$} \\
\hline 19 & 16.89 & $<200$ & $935[\mathrm{M}-\mathrm{H}]^{-}$ & $\mathbf{6 3 3}, 301$ & Galloyl-di-HHDP-glucose & 936 & {$[13,19,20]$} \\
\hline 20 & 17.18 & 376,274 & $1235[\mathrm{M}-\mathrm{H}]^{-}$ & $\mathbf{9 3 3}, 631,469,301$ & di-HHDP-glucose-galloyl-ellagic acid & 1236 & [9] \\
\hline 21 & 17.49 & 272 & $935[\mathrm{M}-\mathrm{H}]^{-}$ & $\mathbf{6 3 3}, 301$ & Galloyl-di-HHDP-glucose & 936 & [13] \\
\hline 22 & 18.00 & 360 & $433[\mathrm{M}-\mathrm{H}]^{-}$ & 301 & Ellagic acid pentoside & 434 & {$[9,20]$} \\
\hline 23 & 18.25 & $350 \mathrm{sh}$ & $535[\mathrm{M}-\mathrm{H}]^{-}$ & 491, 397 & unknown & & [9] \\
\hline 24 & 19.28 & 360 & $895[\mathrm{M}-\mathrm{H}]^{-}$ & $743, \mathbf{5 3 5}, 437,301$ & unknown & & \\
\hline 25 & 19.32 & 360 & $447[\mathrm{M}-\mathrm{H}]^{-}$ & $447, \mathbf{3 0 1}$ & Ellagic acid desoxyhexoside & 448 & [20] \\
\hline 26 & 20.30 & 240 & $1869[\mathrm{M}-\mathrm{H}]^{-}$ & $\begin{array}{l}\mathbf{1 5 6 7}, 1265,1085,935 \\
897,783,633\end{array}$ & Sanguiin H-6 & 1870 & {$[13,19,20]$} \\
\hline 27 & 22.10 & 278 & $935[\mathrm{M}-\mathrm{H}]^{-}$ & $\mathbf{6 3 3}, 301$ & Galloyl-di-HHDP-glucose & 936 & [13] \\
\hline 28 & 22.77 & 368 & $301\left[^{\mathrm{M}-\mathrm{H}}\right]^{-}$ & 301 & Ellagic acid & 302 & {$[9,13,19,20]$} \\
\hline 29 & 24.46 & 351 & $477[\mathrm{M}-\mathrm{H}]^{-}$ & 301 & Quercetin-3-O-glucuronide & 448 & {$[9,13,19,20]$} \\
\hline 30 & 25.79 & 270 & $939[\mathrm{M}-\mathrm{H}]^{-}$ & $787, \mathbf{7 6 9}$ & penta- $O$-Galloylglucoside & 940 & [23] \\
\hline 31 & 26.78 & 282 & $463[\mathrm{M}-\mathrm{H}]^{-}$ & 301 & Quercetin-3-glucoside & 464 & {$[9,13]$} \\
\hline 32 & 26.96 & 282 & $533[\mathrm{M}-\mathrm{H}]^{-}$ & $\mathbf{4 1 3}, 353,232,293$ & unknown & & \\
\hline 33 & 28.40 & 282 & $1108[\mathrm{M}-\mathrm{H}]^{-}$ & $\begin{array}{l}839,751,715,392, \\
\mathbf{3 5 7}, 195\end{array}$ & unknown & & \\
\hline 34 & 28.58 & 283 & $356[\mathrm{M}-\mathrm{H}]^{-}$ & 195 & unknown & & \\
\hline 35 & 29.28 & 277 & $1085[\mathrm{M}-\mathrm{H}]^{-}$ & $\mathbf{7 8 3}, 765,451$ & Ellagitannin & 1086 & \\
\hline 36 & 29.63 & 267 & $447[\mathrm{M}-\mathrm{H}]^{-}$ & 284 & Kaempferol-3-hexoside & 448 & \\
\hline 37 & 30.83 & 268 & $447[\mathrm{M}-\mathrm{H}]^{-}$ & 285 & Kaempferol-3-O-glucoside & 448 & {$[13,20]$} \\
\hline 38 & 34.86 & $335 \mathrm{sh}$ & $533[\mathrm{M}-\mathrm{H}]^{-}$ & 489 & Kaempferol-3-O-malonylglucoside & 534 & [19] \\
\hline 39 & 35.16 & 282 & $593[\mathrm{M}-\mathrm{H}]^{-}$ & 447,285 & Kaempferol-3-O-coumaroylglucoside & 594 & {$[9,13,19,20]$} \\
\hline 40 & 36.36 & $280 \mathrm{sh}$ & $593[\mathrm{M}-\mathrm{H}]^{-}$ & 447,285 & Kaempferol-3-O-coumaroylglucoside & 594 & [26] \\
\hline
\end{tabular}

$\mathrm{sh}=$ shoulder; numbers in bold indicate predominant ions. 

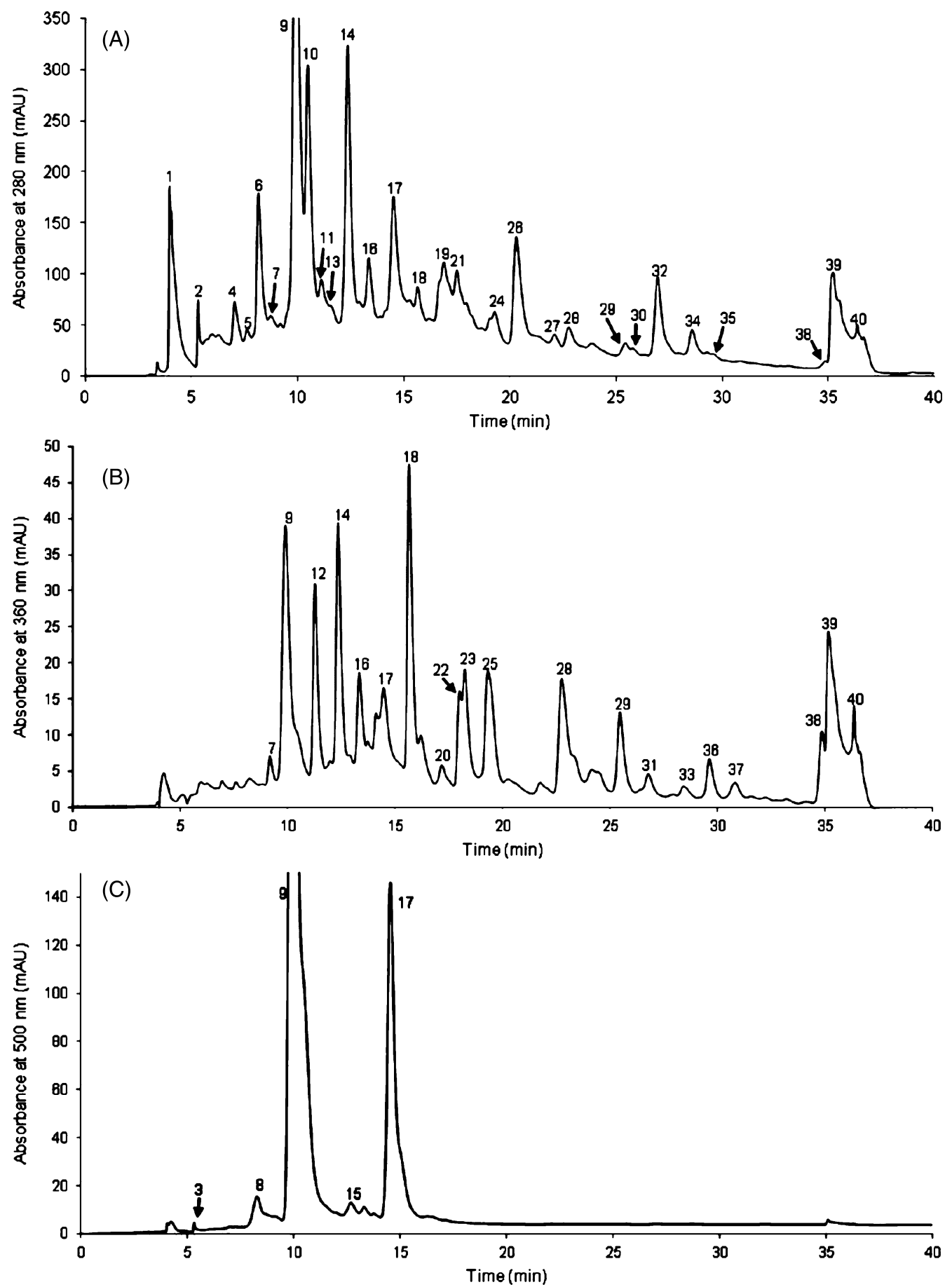

Fig. 1. HPLC chromatograms of strawberry extract at 280 (A), 360 (B) and $500 \mathrm{~nm}$ (C), numbers ascribed to the peaks refer to those in Table 1.

Ellagitannins are esters of hexahydroxydiphenic acid and a polyol, usually glucose [21]. They are called hydrolysable tannins due to their ability to release ellagic acid, which is formed from the hydrolytic release of HHDP ester groups, undergoing spontaneous lactonization [19]. In the chromatographic separation of the SE peaks 4 and 5 originated from isomeric forms of di-HHDP-glucose. The negative molecular ion at a $\mathrm{m} / \mathrm{z}$ of 783 was observed, 
and its fragmentation resulted in ions at a $\mathrm{m} / \mathrm{z}$ of 481 (loss of HHDP, $302 \mathrm{Da}$ ), 301 (loss of HHDP-glucose, $482 \mathrm{Da}$ ) and $275[13,19,20]$. Based on the appearance of a negative molecular ion at a $\mathrm{m} / \mathrm{z} 633$, and fragment ions at $\mathrm{m} / \mathrm{z} 463$ and 301, peak 12 was identified as HHDP-galloyl-glucose [9]. Peaks 19, 21 and 27 were identified as galloyl-di-HHDPglucose isomers. The MW of this compound amounts to 936 , which was reflected in a deprotonated molecular ion at a $\mathrm{m} / \mathrm{z}$ of 935 . During fragmentation ions with $\mathrm{m} / \mathrm{z}$ of 301 and 633 were formed, which corresponds to ellagic acid and galloyl-HHDP-glucose moieties. Peak 20 was identified as di-HHDP-glucose-galloyl-ellagic acid (davuriicin M1) due to a molecular ion at a $\mathrm{m} / \mathrm{z}$ of 1235 , which corresponds to $\mathrm{MW}$ of this compound, and fragmentation pattern $(\mathrm{m} / \mathrm{z} 933,301)$ in accordance with recently published results of Aaby et al. [9]. Peak 26 displayed a negative molecular ion at $\mathrm{m} / \mathrm{z}$ of 1869 . It corresponds to a mass of 1870 , characteristic for the most representative ellagitannin of strawberry, sanguiin H-6 [5], a dimer of galloyl-di-HHDP-glucoside. The fragmentation pattern observed have been already reported: $\mathrm{m} / \mathrm{z}$ of 1567 (loss of HHDP), 1265 (loss of di-HHDP), 935 (loss of galloyl-di-HHDP-glucoside) [20]. However, the identification has not been fully clarified. Vrhovsek et al. [22] supposed that the main ellagitannin in strawberries is agrimoniin, an almost identical structural isomer of sanguiin H-6, with sanguiin being only a minor compound of strawberry. However, taking into consideration the information given by the authors on the elution order of those compounds, we can assume that the questioned peak 26 originates from sanguiin $\mathrm{H}-6$, due to its elution from RP-18 column before ellagic acid [22]. Ions displayed by the compound eluting as a peak 35 were also observed in the fragmentation of sanguiin: a molecular ion at a $m / z$ 1085, and a fragment ion at a $m / z$ of 783 . However, additional fragment ions at $m / z, 765,451$ suggest that it is another ellagitannin. The same molecular ion and fragmentation pattern was noted by Barrajón-Catalán et al. [23] for the compound present in Cistus species and was identified as cornusiin B.

\subsection{Gallotannins}

Peak 30 was identified as penta- $O$-galloyl-glucoside, a compound belonging to the gallotannin family. It has been also detected in the pulp, peels and kernels of mango [24]. The displayed negative molecular ion at a $\mathrm{m} / \mathrm{z}$ of 939 corresponds to MW of penta- $O$-galloyl-glucoside - 940, whereas fragment ions observed in the $\mathrm{MS}^{2}$ at $m / z$ of 787 and 769 result from elimination of one galloyl group (152 Da) or loss of gallic acid (170 Da), respectively.

The presence of above mentioned gallotannin has not been reported in strawberry fruits up to date, however, the analysis of secondary metabolites of strawberry achenes revealed large contents of trigalloyl glucose and pentagalloyl glucose, both diminishing during fruit ripening [25]. At the same time, the increase in ellagitannins content was observed, which confirms that gallotannins are precursors of ellagitannins formation. Therefore, gallotannis in SE might originate from the achenes of the fruits.

\subsection{Hydroxycinnamic acid derivatives}

This group of compounds shows characteristic UV absorbance in the range of 310-330 nm. Peaks 10 and 18 displayed a deprotonated ion at a $m / z$ of 449, and fragment ions at $m / z, 355,325,287$ (loss of hexose), 269, as well as an ion characteristic for ferulic acid - 193. They were identified as isomeric forms of ferulic acid hexose derivatives. However, further identification was not possible. Peak 14 was identified as $p$-coumarylhexose due to the presence of negative molecular ion at a $\mathrm{m} / \mathrm{z}$ of 325 , which during fragmentation displayed fragment ion at a $\mathrm{m} / \mathrm{z}$ of 163 (coumarate residue) and 145 (coumaryl residue).

\subsection{Flavonols}

In accordance with literature data, quercetin and kaempferol derivatives were identified as the major flavonols in strawberries [5]. Peak 29 and 31 were identified as quercetin derivatives due to a characteristic fragment ion at $\mathrm{m} / \mathrm{z}$ of 301 originating from the quercetin moiety. The peak eluting at $24.46 \mathrm{~min}$ was identified as quercetin-3-O-glucuronide since it displayed a negative molecular ion at $\mathrm{m} / \mathrm{z}$ of 477 . The peak eluting at $26.78 \mathrm{~min}$ displayed a negative molecular ion at $\mathrm{m} / \mathrm{z}$ of 463, which corresponds to quercetin-3-O-glucoside. The late eluting peaks 36-40 were designated as kaempferol derivatives according to their UV spectra and MS analysis. Peak 36 was a kaempferol hexoside due to a parental ion at a $m / z$ of 447 and a fragment ion at a $m / z$ of 285 . Peak 37 was identified as kaempferol-3-glucoside. It 
displayed a deprotonated ion at $\mathrm{m} / \mathrm{z}$ of 447 and fragment ion at $\mathrm{m} / \mathrm{z}$ of 285 , corresponding to kaempferol aglycone. Peak 38 was assigned to kaempferol-3-malonylglucoside due to a negative ion at $\mathrm{m} / \mathrm{z}$ of 533 and the presence of fragment ion at $\mathrm{m} / \mathrm{z}$ of 489 resulting probably from decarboxylation [20]. Peak 39 and 40 were probably positional isomers of kaempferol coumarylglucoside. The negative molecular ions and their $\mathrm{MS}^{2}$ fragment ions were identical, only in the UV spectrum little variations were noticed. Similar observation has been recently reported by Simirgiotis et al. [26].

\subsection{Flavanols}

Flavanol monomers and procyanidins are characterized by UV spectra with a $\lambda_{\max }$ at $280 \mathrm{~nm}$. However, they are often overlaid by the other compounds displaying a higher UV response [20]. Peak 11 has a [M-H] ${ }^{-}$at a $m / z$ 577, a main fragment ion at a $\mathrm{m} / \mathrm{z} 425$, and less intense fragment ions at $\mathrm{m} / \mathrm{z} 431,407$ and 289, therefore it was identified as a procyanidin dimer. Peaks 2 and 13 displayed a negative molecular ion at $m / z$ of 865 and $\mathrm{MS}^{2}$ fragment ions at $m / z$ of $739,695,577$, which corresponds to a procyanidin trimer. The compound eluting at 13.34 min was identified as a procyanidin pentamer due to the presence of negative molecular ion at $\mathrm{m} / \mathrm{z} 1441$, and fragment ions at $\mathrm{m} / \mathrm{z}, 1315$, 865, and 575, which have been previously reported by Aaby et al. [9].

\subsection{Other compounds}

Peak 1 was identified as ascorbic acid that, due to its strong hydrophilicity, elutes very early from a reversed phase column. It was characterized by a negative molecular ion at a $\mathrm{m} / \mathrm{z}$ of 175 , and a fragment ion at a $\mathrm{m} / \mathrm{z}$ of 115 . Peak 6 exhibited a strong UV absorption at $279 \mathrm{~nm}$, and displayed a molecular ion at $\mathrm{m} / \mathrm{z}$ of 205 in the positive mode, and a fragment ion at a $\mathrm{m} / \mathrm{z}$ of 188 . The presence of a compound with the same mass spectra characteristic was previously reported by Aaby et al. [20]. However, the authors designated the compound as unknown. On the basis of UV and MS spectra we assumed that the compound might be tryptophan. A comparison with the UV spectra and RT of the standard compound enabled unambiguous identification of this compound as tryptophan.

\subsection{Non-identified compounds}

Some compounds from SE of Charlotte cultivar remain to be unambiguously identified. The fragmentation pattern of peak 7 with a $\mathrm{m} / \mathrm{z}$ of 1917 does not match any literature known substance. Peak 23 could not be assigned either. Aaby et al. [9] reported similar problems with identification of the substance strongly absorbing at $360 \mathrm{~nm}$, not visible at $280 \mathrm{~nm}$, showing the same fragmentation pattern. According to the deprotonated ion at a $\mathrm{m} / \mathrm{z}$ of 895 and the UV maxima at $360 \mathrm{~nm}$, peak 24 could be assigned as an ellagic acid rhamnoside, which is detected as singly charged dimer $[2 \mathrm{M}-\mathrm{H}]^{-}$. However, the fragments did not match those reported for this compound in the literature [26]. Peaks 32,33 and 34 escaped identification.

\section{Conclusions}

In general, the phenolic profile of Charlotte cultivar strawberry fruits resembles those reported for numbers of other strawberry cultivars. However, some peculiarities of the cultivar in terms of phenolic composition can be pointed out. The fruits contained phenolics belonging to six groups: anthocyanins, ellagic acid and its conjugates, gallotannins, flavonols, flavanols and hydroxycinnamic acid derivatives, from which pentagalloyl glucose was identified in strawberry fruits for the first time. On the other hand, the fruits of the analysed cultivar lacked catechin, present in previously investigated strawberry cultivars.

\section{Acknowledgments}

Sascha Diering was financially supported by the ERASMUS traineeship program of the European Union. Agnieszka Kosińska was a fellow of the Scientific Exchange Programme founded by Rectors' Conference of the Swiss 
Universities (CRUS, Project code: 10.127). The authors are very grateful to Dr. Christoph Carlen (Agroscope Changins-Wädenswil ACW Research Centre Conthey) for a kind gift of strawberry fruits.

\section{References}

[1] Paredes-López O, Cervantes-Ceja ML, Vigna-Pérez M, Hernández-Pérez T. Berries: Improving human health and healthy aging, and promoting quality life-A review. Plant Foods Hum Nutr. 2010;65:299-308.

[2] Da Silva Pinto M, Lajolo FM, Genovese MI. Bioactive compounds and antioxidant capacity of strawberry jams. Plant Foods Hum Nutr. 2007;62:127-31.

[3] Singh M, Arseneault M, Sanderson T, Murthy V, Ramassamy C. Challenges for research on polyphenols from foods in Alzheimer's disease: Bioavailability, metabolism, and cellular and molecular mechanisms. J Agric Food Chem. 2008;56:4855-73.

[4] Kylli P, Nohynek L, Puupponen-Pimiä R, Westerlund-Wikström B, Leppänen T, Welling J, et al. Lingonberry(Vaccinium vitis-idaea) and European cranberry(Vaccinium microcarpon) proanthocyanidins: Isolation, identification, and bioactivities. J Agric Food Chem. 2011;59:3373-84.

[5] Giampieri F, Tulipani S, Alvarez-Suarez JM, Quiles JL, Mezzetti B, Battino M. The strawberry: Composition, nutritional quality, and impact on human health. Nutr. 2012;28:9-19.

[6] Seeram NP. Berry fruits for cancer prevention: Current status and future prospects. J Agric Food Chem. 2008;56:630-35.

[7] Seeram NP, Adams LS, Zhang Y, Lee R, Sand D, Scheuller HS, et al. Blackberry, black raspberry, blueberry, cranberry, red raspberry, and strawberry extracts inhibit growth and stimulate apoptosis of human cancer cells in vitro. J Agric Food Chem. 2006;54:9329-39.

[8] Pincemail J, Kevers C, Tabart J, Defraigne JO, Dommes J. Cultivars, culture conditions and harvest time influence phenolic and ascorbic acid contents and antioxidant capacity of strawberry (Fragaria x ananassa). J Food Sci. 2012;77:205-10.

[9] Aaby K, Mazur S, Nes A, Skrede G. Phenolic compounds in strawberry (Fragaria x ananassa Duch.) fruits: Composition in 27 cultivars and changes during ripening. Food Chem. 2012;132:86-97.

[10] Buendía B, Gil MI, Tudela JA, Gady AL, Medina JJ, Soria C, et al. HPLC-MS analysis of proanthocyanidin oligomers and other phenolics in 15 strawberry cultivars. J Agric Food Chem. 2010;58:3916-26.

[11] Lopes da Silva F, Escribano-Bailón MT, Pérez Alonso JJ, Rivas-Gonzalo JC, Santos-Buelga C. Anthocyanin pigments in strawberry. LWT. 2007;40:374-82.

[12] Kelebek H, Selli S. Characterization of phenolic compounds in strawberry fruits by RP-HPLC-DAD and investigation of their antioxidant capacity. J Liquid Chromat Rel Technol. 2011;34:2495-504.

[13] Kajdžanoska M, Gjamovski V, Stefova M. HPLC-DAD-ESI-MS ${ }^{\text {n }}$ identification of phenolic compounds in cultivated strawberries from Macedonia. Maced J Chem Chem Eng. 2010;29:181-94.

[14] Josuttis M, Carlen C, Crespo P, Nestby R, Toldam-Andersen TB, Dietrich H, et al. A comparison of bioactive compounds of strawberry fruit from Europe affected by genotype and latitude. J Berry Res. 2012;2:73-95.

[15] Cheplick S, Kwon YI, Bhowmik P, Shetty K. Phenolic-linked variation in strawberry cultivars for potential dietary management of hyperglycemia and related complications of hypertension. Biores Techn. 2010;101:404-13.

[16] Vandendriessche T, Vermeir S, Mayayo Martinez C, Hendrickx Y, Lammertyn J, Nicolaï BM, et al. Effect of ripening and inter-cultivar differences on strawberry quality. LWT. 2012; doi:10.1016/j.lwt.2011.12.037

[17] Ciref Création Variétale Charlotte variety. http://www ciref fr/Charlotte html. Accessed 05 May 2012.

[18] Kosińska A, Xie Y, Diering S, Heritier J, Andlauer W. Stability of phenolic compounds isolated from cocoa, green tea and strawberries in Hank's balanced salt solution under cell culture conditions. Pol J Food Nutr Sci. 2012;62:91-6.

[19] Seeram NP, Lee R, Scheuller HS, Heber D. Identification of phenolic compounds in strawberries by liquid chromatography electrospray ionization mass spectroscopy. Food Chem. 2006;97:1-11.

[20] Aaby K, Ekeberg D, Skrede G. Characterization of phenolic compounds in strawberry (Fragaria $x$ ananassa) fruits by different HPLC detectors and contribution of individual compounds to total antioxidant capacity. J Agric Food Chem. 2007;53:4395-406.

[21] Okuda T, Yoshida T, Hatano T, Ito H. Ellagitannins renewed the concept of tannins. In Quideau S (ed) Chemistry and Biology of Ellagitannins - An Underestimated Class of Bioactive Plant Polyphenols, World Scientific Publishing Company, 2011; pp. 1-54.

[22] Vrhovsek U, Guella G, Gasperotti M, Pojer E, Zancato M, Mattivi F. Clarifying the identity of the main ellagitannin in the fruit of the strawberry, Fragaria vesca and Fragaria ananassa Duch. J Agric Food Chem. 2012;60:2507-16.

[23] Barrajón-Catalán E, Fernández-Arroyo S, Roldán C, Guillén E, Saura D, Segura-Carretero A, et al. A systematic study of the polyphenolic composition of aqueous extracts deriving from several Cistus genus species: Evolutionary relationship. Phytochem Anal. 2011;22:303-12.

[24] Berardini N, Carle R, Schieber A. Characterization of gallotannins and benzophenone derivatives from mango (Mangifera indica L. cv. 'Tommy Atkins') peels, pulp and kernels by high-performance liquid chromatography/electrospray ionization mass spectrometry. Rapid Commun Mass Spectrom. 2004;18:2208-16.

[25] Fait A, Hanhineva K, Beleggia R, Dai N, Rogachev I, Nikiforova VJ, et al. Reconfiguration of the achene and receptacle metabolic networks during strawberry fruit development. Plant Physiol. 2008;148:730-50. 
[26] Simirgiotis MJ, Schmeda-Hirschmann G. Determination of phenolic composition and antioxidant activity in fruits, rhizomes and leaves of the white strawberry (Fragaria chiloensis spp. chiloensis form chiloensis) using HPLC-DAD-ESI-MS and free radical quenching techniques. J Food Comp Anal. 2010;23:545-53.

[27] Cheel J, Theoduloz C, Rodríguez J, Saud G, Caligari PDS, Schmeda-Hirschmann G. E-Cinnamic acid derivatives and phenolics from Chilean strawberry fruits, Fragaria chiloensis ssp. chiloensis. J Agric Food Chem. 2005;53:8512-8. 